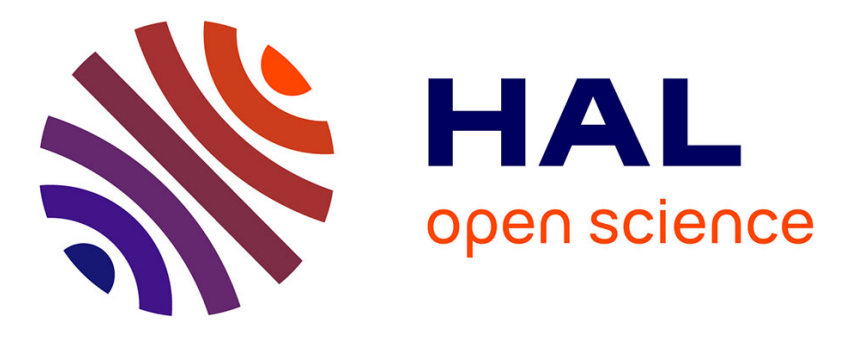

\title{
Ion dynamics in a linear radio-frequency trap with a single cooling laser
}

\author{
Mathieu Marciante, Caroline Champenois, Annette Calisti, Jofre \\ Pedregosa-Gutierrez, Martina Knoop
}

\section{To cite this version:}

Mathieu Marciante, Caroline Champenois, Annette Calisti, Jofre Pedregosa-Gutierrez, Martina Knoop. Ion dynamics in a linear radio-frequency trap with a single cooling laser. Physical Review A : Atomic, molecular, and optical physics [1990-2015], 2010, 82, pp.033406. 10.1103/PhysRevA.82.033406 . hal-00476701v2

\section{HAL Id: hal-00476701 \\ https://hal.science/hal-00476701v2}

Submitted on 10 Sep 2010

HAL is a multi-disciplinary open access archive for the deposit and dissemination of scientific research documents, whether they are published or not. The documents may come from teaching and research institutions in France or abroad, or from public or private research centers.
L'archive ouverte pluridisciplinaire HAL, est destinée au dépôt et à la diffusion de documents scientifiques de niveau recherche, publiés ou non, émanant des établissements d'enseignement et de recherche français ou étrangers, des laboratoires publics ou privés. 


\title{
Ion dynamics in a linear radio-frequency trap with a single cooling laser
}

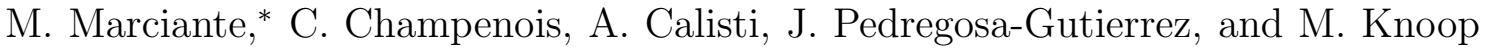 \\ Physique des Interactions Ioniques et Moléculaires, \\ UMR 6633 CNRS et Aix-Marseille Université, \\ Centre de Saint Jérôme, Case C21, 13397 Marseille Cedex 20, France
}

(Dated: September 10, 2010)

\begin{abstract}
We analyse the possibility of cooling ions with a single laser beam, due to the coupling between the three components of their motion induced by the Coulomb interaction. For this purpose, we numerically study the dynamics of ion clouds of up to 140 particles, trapped in a linear quadrupole potential and cooled with a laser beam propagating in the radial plane. We use Molecular Dynamics simulations and model the laser cooling by a stochastic process. For each component of the motion, we systematically study the dependence of the temperature with the anisotropy of the trapping potential. Results obtained using the full radio-frequency (rf) potential are compared to those of the corresponding pseudo-potential. In the rf case, the rotation symmetry of the potential has to be broken to keep ions inside the trap. Then, as for the pseudo-potential case, we show that the efficiency of the Coulomb coupling to thermalize the components of motion depends on the geometrical configuration of the cloud. Coulomb coupling appears to be not efficient when the ions organise as a line or a pancake and the three components of motion reach the same temperature only if the cloud extends in three dimensions.
\end{abstract}

PACS numbers: 37.10.Rs; 37.10.Vz; 37.10.Ty

*Electronic address: mathieu.marciante@etu.univ-provence.fr 


\section{INTRODUCTION}

The advent of laser cooling [1, 2] generalized infinite trapping times in radio-frequency (rf) traps [3] and allowed observing a single atom by its emitted fluorescence [4]. Doppler cooling in rf traps only employs a single laser beam, as the trapping potential brings back the atom into resonance when it is counter-propagating with the laser beam. Although heating is expected perpendicularly to the laser beam from the mechanical effect of light [5], several experiments with single ions in spherical Paul traps have demonstrated cooling to the Doppler limit with a single laser beam, as long as it does not propagate along the symmetry axis of the trap [6] 8 ]. This is attributed to trap defects which induce a coupling between the three components of the motion [6, 9]. For ions in a linear quadrupole trap, the use of a single laser beam to completely cool a trapped sample in its three degrees of freedom remains an open question which we address by numerical simulations in the present work. Coulomb repulsion between the ions is expected to couple their motion and to result in the thermalization of the three degrees of freedom, independently of the direction of the laser beam. Nevertheless, depending on the aspect ratio of the cloud, the coupling may be more or less efficient. The dependance of this aspect ratio with the anisotropy of the confining pseudo-potential, as well as the transitions between different organized structures have been studied numerically by J. Schiffer in [10] and analytically by D. Dubin [11]. These works show that the minimal energy configurations can be one, two or three dimensional, depending on the aspect ratio of the potential and the number of trapped ions. In the present work, we numerically study the thermalization of the different degrees of motion under Doppler laser cooling by Molecular Dynamics methods. The study is carried out for different aspect ratios and ion numbers, in order to determine the influence of the cloud anisotropy on the cooling of the sample. In the simulations, Doppler laser cooling is introduced by the mechanical effect of absorption and emission of photons by the atoms.

Laser cooling of an ion string has been previously studied analytically using the common vibrational mode to describe the motion of the ions 12 14. In these works, the string is assumed to be tightly bound in the radial plane and the only considered motion is the one along the trap axis. Contrary to our configuration, this motion is directly laser cooled and its thermalization with the motion in the radial plane is not addressed in these works. Studies on ion strings [14] and more generally on ion crystals [15] have shown that the motion along 


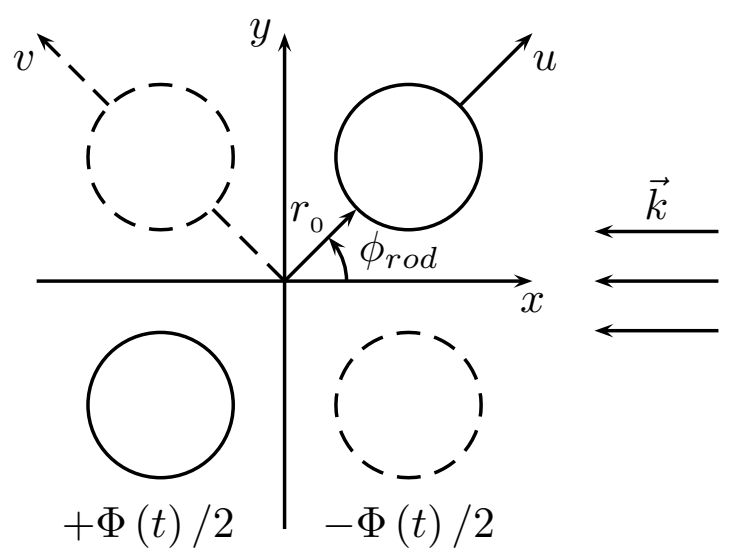

FIG. 1: Geometry of the trap in the $(x, y)$-plane. $\vec{k}$ is the laser wave-vector. A voltage $\pm \Phi(t) / 2$ is applied on each pair of opposite rods.

one direction is cooled provided the cooling laser has a projection along it. The role of the rf-driven motion on the cooling efficiency has been analyzed for crystals [16] as well as for single ions [17], pointing the influence of the laser detuning compared to the rf frequency.

The present article is organized as follows, we first introduce the studied system and the numerical method used. Then, in the following section, we study the effect of the anisotropy of the cloud on the cooling, in the adiabatic approximation where the rf trapping field is modeled by a static pseudo-potential. In the last section, the same properties are analyzed in the case where the full rf electric field is introduced to trap the ions.

\section{FRAMEWORK}

The system under study is an ensemble of $N$ ions of mass $m$ and charge $q_{e}=+e$, confined in a linear quadrupole trap and Doppler laser cooled by a single laser beam of wave-vector $\vec{k}$. The trapping field is represented either by the time dependent electric field (the rf-case) or by the gradient deduced from the static pseudo-potential (pseudo-potential case), associated to the rf electric field in the adiabatic approximation [3, 22]. We assume that the laser beam intensity is uniform over the area covered by the ions. The $x$ axis is always defined by the propagation of the cooling laser beam and a $(u, v)$ axis system is related to the trap electrodes. An eventual trap rotation in the $(x, y)$ plane with respect to the laser beam is described by the angle $\phi_{\text {rod }}$ (see Fig. 1). 


\section{A. Dynamics}

In this section, we explicitly write the equations for the forces acting on each ion. They result from the trapping potential, the Coulomb repulsion between ions and the absorptionemission process implied in the Doppler laser cooling. The rf electric field assures trapping in the radial plane of the trap. To achieve trapping along the symmetry axis ( $z$ axis), a static voltage is applied to additional electrodes at both ends of the trap. In the center of the trap, the created potential can be considered as harmonic [18] and characterized by the oscillation frequency $\omega_{z}$. The full trapping potential $U_{t r p}$ is:

$$
\begin{aligned}
U_{t r p}= & \frac{q_{e} \Phi(t)}{2 r_{0}^{2}}\left[\left(x^{2}-y^{2}\right) \cos \left(2 \phi_{\text {rod }}\right)+2 x y \sin \left(2 \phi_{r o d}\right)\right] \\
& -\frac{m \omega_{z}^{2}}{2}\left(\frac{x^{2}+y^{2}}{2}-z^{2}\right)
\end{aligned}
$$

with $r_{0}$ the inner radius of the trap and $\Phi(t)=V_{d c}-V_{0} \cos \left(\Omega_{r f} t\right)$ the potential difference applied between neighboring electrodes. The motion of a single ion due to the quadrupole electric field is characterized by the Mathieu parameters [19, 20] which depend on the experimental parameters like:

$$
\begin{aligned}
q_{u} & =\frac{2 q_{e} V_{0}}{m r_{0}^{2} \Omega_{r f}^{2}}, \\
a_{u} & =\frac{4 q_{e} V_{d c}}{m r_{0}^{2} \Omega_{r f}^{2}} .
\end{aligned}
$$

The radial contribution of the harmonic static potential (see Eq. 1) can be taken into account by an effective Mathieu parameter [21], denoted $\Delta a$ and which can be expressed like $\Delta a=$ $2 \omega_{z}^{2} / \Omega_{r f}^{2}$. These parameters define the oscillation frequencies of an ion in the radial plane of the trap. In the adiabatic approximation [22], the dynamics of one ion is the superposition of a harmonic motion (called macro-motion) and the rf-driven motion (called micro-motion). The harmonic contribution is derived from the pseudo-potential $\Psi_{t r p}$, expressed in the frame $(u, v)$ as:

$$
\Psi_{t r p}=\frac{1}{2} m \omega_{r+}^{2} u^{2}+\frac{1}{2} m \omega_{r-}^{2} v^{2}+\frac{1}{2} m \omega_{z}^{2} z^{2} .
$$

For $\left(\left|a_{u}\right|, q_{u}^{2} / 2\right) \ll 1$ (and in practice $q_{u}<0.4$ ), the oscillation frequencies can be written as

$$
\omega_{r \pm}=\frac{\Omega_{r f}}{2} \sqrt{\frac{q_{u}^{2}}{2} \pm a_{u}-\Delta a}
$$


In the case where no static voltage $V_{d c}$ is applied to the quadrupole rods, $\omega_{r+}=\omega_{r-}$, and the anisotropy of the potential can be characterized by the ratio of its strengths [10]. When the cylindrical symmetry is broken by a static voltage $V_{d c}$ applied to the rf rods (for $V_{d c}>0$, $\left.\omega_{r+}>\omega_{r-}\right)$, it has been shown [10] that the ion structure aligns along the minimal steepness direction $v$. Therefore, we consider that $\omega_{r-}$ becomes the relevant parameter to describe the anisotropy of the trap:

$$
\alpha=\left(\omega_{z} / \omega_{r-}\right)^{2}
$$

The total Coulomb repulsion on one ion $i$ at position $\vec{r}_{i}$ is the sum over the $(N-1)$ other ions of the Coulomb inter-particle force:

$$
\vec{F}_{C_{i}}=\sum_{j \neq i} \frac{q_{e}^{2}}{4 \pi \varepsilon_{0}} \frac{\left(\vec{r}_{i}-\vec{r}_{j}\right)}{\left|\vec{r}_{i}-\vec{r}_{j}\right|^{3}}
$$

Doppler laser cooling is simulated by kicks on the velocity of each ion for each absorption and emission process [23]. On absorption, the momentum transferred to the ion is the momentum of a photon from the laser beam $\hbar \vec{k}=-\hbar k \hat{x}$ (the laser propagation being along $-x$, see Fig. 1). The velocity recoil associated to the emission of one photon is uniformly distributed on a sphere of radius $\hbar k / m$. For a single emission event, the direction of the recoil is randomly distributed by the use of equation

$$
\overrightarrow{\delta v_{e m}}=\frac{\hbar k}{m}\left(\sqrt{\theta_{1}\left(1-\theta_{1}\right)}\left[\cos \left(\theta_{2}\right) \hat{x}+\sin \left(\theta_{2}\right) \hat{y}\right]+\left(1-2 \theta_{1}\right) \hat{z}\right)
$$

where $\theta_{1}$ and $\theta_{2}$ are stochastic variables uniformly distributed between 0 and 1 . To calculate the probability of absorption on the cooling transition, the ion is considered as a two level system, characterized by the spontaneous emission rate of the excited level $\Gamma_{0}$, far larger than the recoil frequency $\hbar k^{2} /(2 m)$. The strength of the laser-atom coupling is defined by the Rabi frequency $\Omega_{r}$. The relevant time scale of the laser-atom interaction is of the same order of magnitude as the integration time step. Nevertheless, we neglect the time correlation effect between the motion and the laser-atom interaction and assume that for each time step, the stationary internal state is reached. In the frame of these assumptions, the probability $P_{a b s}(t)$ for an ion in the ground state with velocity $\vec{v}(t)$ to absorb a photon during the time step $\Delta t$ is given by

$$
P_{a b s}=\frac{\Gamma_{0} \Delta t}{2} \frac{\Omega_{r}^{2} / 2}{\left(\delta_{l}-\vec{k} \cdot \vec{v}(t)\right)^{2}+\left(\Gamma_{0} / 2\right)^{2}+\Omega_{r}^{2} / 2}
$$


and only depends on the velocity of the ion by the Doppler shift, $\delta_{l}$ being the laser detuning for an ion at rest. The stimulated emission is neglected and the probability $P_{e m}$ for an ion in the excited state to spontaneously emit a photon during the time step $\Delta t$ follows the usual decay law $P_{e m}=\Gamma_{0} \Delta t$. This model for Doppler laser cooling implies that the numerical integration keeps track of the internal state of each ion. At each time step, the $N$ ions can change their internal state. The process is controlled individually by the comparison between a uniform random number associated to a single ion and the absorption probability if the ion is in the ground state or to the emission probability otherwise.

One of the parameters characterizing the evolution of the system is its temperature. As we are interested in the coupling between the three components of motion, we do not assume a uniform temperature for the three axes and define a temperature for each of them $T_{k}$, with $k=x, y, z$. As the rf driven motion (or micromotion) does not contribute to the temperature [24 26], our definition of the temperature uses the part of the velocity which does not show the rf periodicity:

$$
T_{k}=\frac{1}{k_{B}} m\left(\left\langle{\overline{v_{k}^{2}}}_{k}\right\rangle-\left\langle\bar{v}_{k}\right\rangle^{2}\right)
$$

where the overline holds for the average over one rf period. The $<>$-brackets are the statistical averages over the number of ions. This definition also subtracts a possible motion of the center of mass (COM).

\section{B. Numerical issues}

The time evolution of a system made of $N=35,70$ and 140 trapped ions is studied via Molecular Dynamics simulations. We use the velocity-Verlet algorithm for the integration of the equations of motion and the time step is taken as 1/100 of the rf-period. For all cases studied in the following, the radio-frequency trapping field is kept constant with a frequency $\Omega_{r f} / 2 \pi=10 \mathrm{MHz}$, which results in a time step of $10^{-9} \mathrm{~s}$, and an rf amplitude $V_{0}=960 \mathrm{~V}$ in a linear trap of inner radius $r_{0}=2.5 \mathrm{~mm}$. The trapped ion species is the most abundant calcium isotope $(m=40 \mathrm{amu})$, and for this species, the rf trapping parameters lead to a Mathieu parameter $q=0.187$, which obeys the validity criteria of the adiabatic approximation. The aspect ratio $\alpha$ of the potential is changed by tuning $\omega_{z}$ which takes values between $2 \pi \times 3.5 \mathrm{kHz}$ and $2 \pi \times 1.15 \mathrm{MHz}$. For Doppler laser cooling, we use the strong dipole transition of calcium ion at $397 \mathrm{~nm}$, which has a natural linewidth of 
$\Gamma_{0}=1.43 \times 10^{8} \mathrm{~s}^{-1}$. To optimize laser cooling [27, 28], the laser detuning is set at half the natural linewidth $\delta_{l}=-\Gamma_{0} / 2$.

The choice for the Rabi frequency results from a compromise between reaching the lowest temperature, which requires $\Omega_{r} \ll \Gamma_{0}$ [27, 28] and keeping a high enough photon scattering rate to counteract the heating induced by the trapping potential, called rf-heating [26, 2931. This heating is induced by the transfer of energy from the rf power source to the macro-motion kinetic energy because of non-linear resonances [32, 33]. These resonances can be induced by higher order contributions in the rf trapping potential (not included in the simulations) and by the Coulomb repulsion between ions [34]. With our trapping configuration, the cooling of the simulated trajectories requires a Rabi frequency of the order of $\Gamma_{0}$ to counteract rf-heating.

As the rf-heating rate depends on the temperature of the sample [26, 31], it is possible to reach lower equilibrium temperatures by varying the Rabi frequency during the cooling process. Indeed, one could imagine starting the experiment with a high Rabi frequency to ensure a high enough scattering rate to reach a temperature for which the rf-heating is negligible [31]. Then, in a second step, the Rabi frequency could be decreased to reach a lower equilibrium temperature. In the present work, we use the same constant Rabi frequency $\Omega_{r}=\Gamma_{0}$ throughout our study in order to focus on the differences in the Coulomb coupling induced by the anisotropy of the cloud. The limit temperatures reached by the cooling process control the amplitude of the oscillation of the ions around their equilibrium position (the non-driven one). This has an effect on the Coulomb coupling through the relative positions of the ions. Slightly different results are then expected with another Rabi frequency, but the general behaviour of the coupling efficiency should not fundamentally change.

Initial conditions are built in an identical way for the rf case and the pseudo-potential case associated to the same rf potential. The aim is to prepare a set of ions in an equilibrium state characterized by a $1 \mathrm{~K}$ temperature. For this purpose, a random position and velocity is given to each ion which evolves under the pseudo-potential trapping force, the Coulomb forces and the photon absorption/emission momentum kicks. The cooling laser is already applied during the preparation of the initial state to avoid a shift in position induced by the radiation pressure at the beginning of the simulations. To prepare an initial equilibrium state at $1 \mathrm{~K}$, a Gaussian thermostat 35 37] is used, which consists in scaling each velocity 
at the end of a fixed period. Starting from these initial conditions, the cloud is then ready to evolve under the Molecular Dynamics simulations which take place in the rf electric field or its associated pseudo-potential. The conversion of the pseudo-potential into its rf counterpart is associated to a rapid rise of the temperature. This discontinuity does not affect the long term equilibrium of the dynamics [24]. The time evolution starts by a cooling phase, which lasts less than $10 \mathrm{~ms}$ and takes the system from the initial $1 \mathrm{~K}$ to temperatures of the order of few mK. Then the dynamics becomes stationary and the system can be characterized. To reach a good precision, each temperature ratio curve results from the average over at least 15 runs with different initial conditions. Error bars are evaluated at $1 \sigma$ deviation from the mean value.

\section{IN THE PSEUDO-POTENTIAL APPROXIMATION}

The structural phase transitions between the minimum energy configurations calculated for different $\alpha$ have been identified by J.P. Schiffer in [10]. The Molecular Dynamics simulations used in that work do not imply any cooling but a thermalization of the sample by a thermostat. Our Doppler laser cooling simulations do not allow to reach these low temperatures and are limited by the Doppler cooling limit $(0.5 \mathrm{mK}$ in the case of calcium ions). Therefore, we observe large thermal fluctuations compared to the configurations demonstrated in [10]. Nevertheless, we refer to J.P. Schiffer's work to look for correlations between the geometric configuration of the set of ions and the efficiency of the Coulomb coupling to cool the degrees of freedom that are not directly laser cooled.

As an illustration of the geometrical evolution of these configurations, let us consider a 70 ion system at very low temperature. For $\alpha<1.5 \times 10^{-3}$, the minimum energy configuration is a linear structure which becomes a planar zig-zag for higher $\alpha$ values [38, until $\alpha \simeq$ $3.1 \times 10^{-3}$. Increasing $\alpha$ beyond this value results in a twisting of the zig-zag, which forms then a 3D structure. It has been shown [10 that the twisting angle increases with increasing $\alpha$. For large enough $\alpha(\alpha \simeq 10)$, the configuration is again a 2D structure, this time in the plane orthogonal to the symmetry axis of the trap. In our simulations, we observe some differences in the equilibrium configurations due to thermal fluctuations. For example, line or zig-zag configurations can only be observed by time-averaging of ion positions. Besides these fluctuation effects we find the same structural transitions between different configurations 
as observed in $[10$.

\section{A. In a potential with cylindrical symmetry}

In this part, we study the cooling of the three degrees of freedom of ions trapped in the pseudo-potential without a static voltage applied on the quadrupole rods $\left(a_{u}=0\right)$ and with a single laser beam propagating along $-x$. The values of $\alpha$ for which the structural transitions occur are in good agreement with those given in [10]. In the cases where the ions form a line along the $z$ axis, there is a large amplitude of motion in the plane orthogonal to the laser beam, showing that laser cooling is not efficient in this plane. A simulated CCD-image of a 70-ion system is shown on Fig. 2 resulting from the projection on two different planes: the top view shows a picture projected on the $(x, z)$ plane which includes the laser propagation axis, the bottom view is a projection on the $(y, z)$ plane, orthogonal to the laser axis. These simulated images show that the thermal fluctuations can be observed experimentally in the direction perpendicular to the trap axis and to the laser beam. A precise analysis of the data reveals that the amplitude of motion in the directions perpendicular to the laser beam is not only due to thermal motion around the COM but also to the motion of the COM itself. The non-cooling of the COM motion in the plane orthogonal to the laser beam has already been analyzed in [39]. Indeed, the COM motion is decoupled from the internal Coulomb forces of the cloud. Hence, the COM behaves like a single particle inside the trap potential. Its kinetic energy is only reduced on the eigen-axis of the trap on which the laser beam has a projection. In the cylindrically symmetric pseudo-potential, the radial directions are all equivalent and the kinetic energy of the COM is reduced only along the laser beam direction.

The dependance of the temperature ratio reached by laser cooling with the anisotropy of the trapping potential is summarised in Fig. 3. For small values of $\alpha$, corresponding to a string of ions, the Coulomb coupling does not lead to cooling in the $y$ and $z$ direction and the temperature ratio shown on Fig. 3 can be as high as 200 . The temperature $T_{x}$ itself fluctuates around the Doppler cooling limit $(0.5 \mathrm{mK})$.

Increasing $\alpha$ leads to the zig-zag structure identified for very low temperatures, but in our case, the amplitude of the thermal motion is comparable to the distance between the two arms of the zig-zag. Moreover, there is no configuration in the $(x, y)$ plane with minimal energy and the calculated structure undergoes stochastic rotations around the $z$-axis, which 


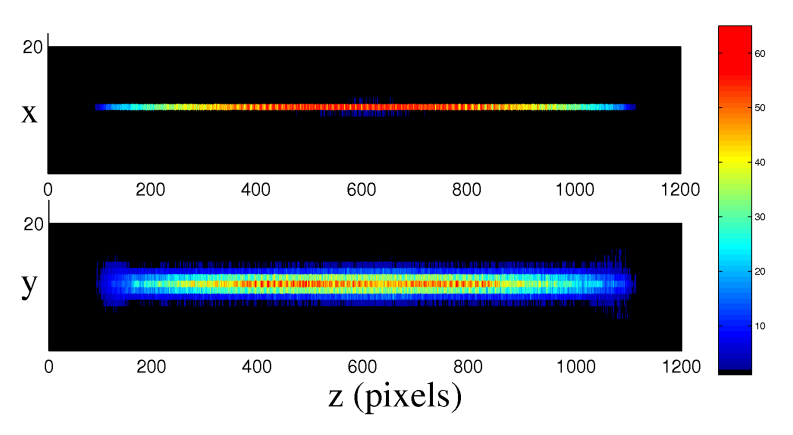

FIG. 2: (Color online) Simulated CCD-image of 70 ions laser cooled along the $x$-axis and trapped in the pseudo-potential associated to the rf field defined in IIB, with an anisotropy characterized by $\alpha=10^{-3}$. The pixel size is $1 \mu \mathrm{m}$ and the signal associated to one pixel is proportional to the number of scattered photons in this pixel, integrated over $10 \mathrm{~ms}$. The picture is projected on the $(x, z)$ plane (top) and on the $(y, z)$ plane (bottom).

prevent from obtaining an averaged structure comparable to the one shown on Fig. 2. The extension of the ion configuration in 3D results in a better coupling between the 3 degrees of freedom which is visible on Fig. 3 with temperature ratios close to 1 . One can notice that the temperature ratio decreases drastically by two orders of magnitude for $\alpha$ values in the range for which the stable configurations at low temperature switch from a line to a twisted zig-zag. When $\alpha$ reaches values for which the ions settle in a plane configuration orthogonal to the trap axis ( $\alpha \geq 10$ for 70 ions), the spatial extension of the cloud in the $z$ direction is too small to keep an efficient coupling between the motion along $z$ and the motion in the $(x, y)$ plane. This translates into a rapid growth of the $T_{z} / T_{x}$ ratio whereas the $T_{y} / T_{x}$ ratio remains very close to 1 (see Fig. 2). The stationary regime is reached when the amplitude of the thermal motion along $z$ is large enough to recover a strong enough coupling with the laser cooled direction of motion. The correlations between the 3D geometric extension of the cloud with the temperature ratio demonstrate the limits of Coulomb coupling for efficient cooling in 3D with a single laser beam.

\section{B. Without cylindrical symmetry}

In this part, we break the cylindrical symmetry of the trapping pseudo-potential by a very small amount $\left(a_{u}=7.805 \cdot 10^{-3}\right.$ or $\left.V_{d c}=20 \mathrm{~V}\right)$ to analyze the effect of the potential 

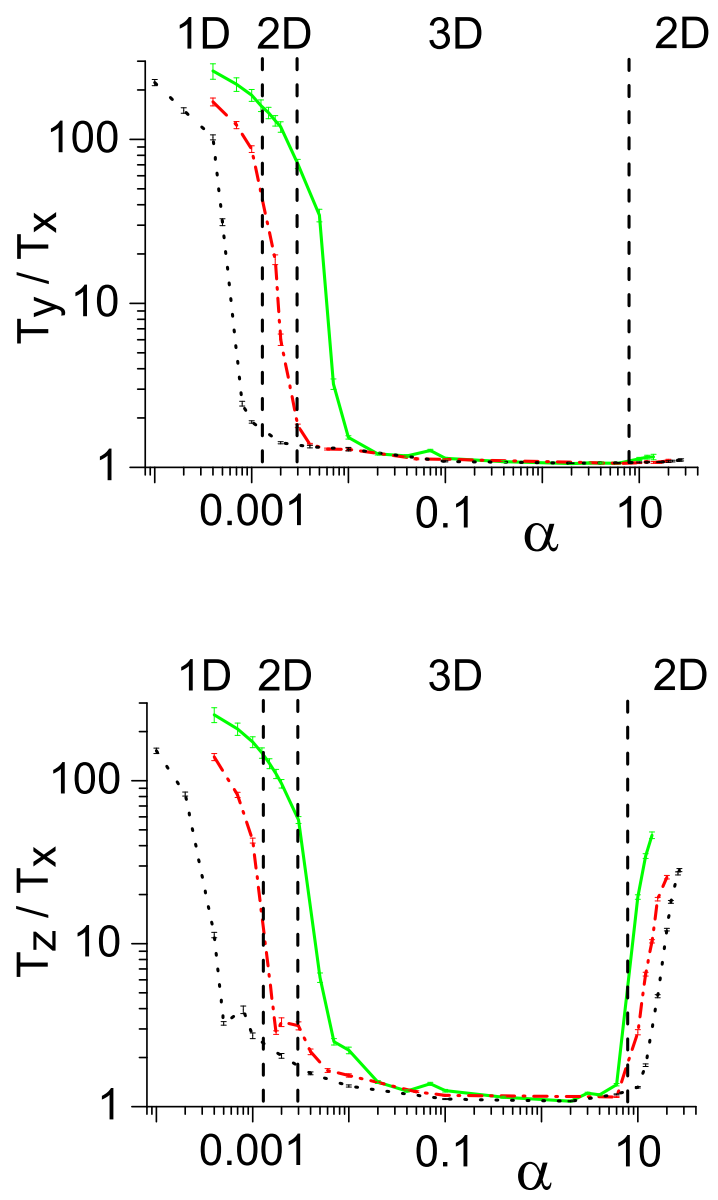

FIG. 3: (Color online) Ratio of the temperature in $y$ (top) or $z$ (bottom) direction with respect to the temperature in the laser cooled direction, versus the anisotropy of the trap $\alpha$. The trapping potential is the static pseudo-potential with cylindrical symmetry $\left(a_{u}=0\right)$. Calculations are made for 35 ions (solid line), 70 ions (dash-dotted line) and 140 ions (dotted line). The vertical lines mark the transition between different geometric configurations for 70 ions.

asymmetry on the cooling efficiency without notably modifying the potential depths. As before, we choose a set of 70 ions as a representative case for the descriptions of the dynamics. For $\alpha \leq 1.5 \times 10^{-3}$, the structure still forms a line along the $z$ direction. But for larger $\alpha$ values, the dynamics in the radial plane is very different from the dynamics in a cylindrical symmetric potential. Once cooled, the ions tend to align in the direction of minimum steepness of the potential, noted $v$ for a positive $V_{d c}$. For $\alpha=2 \times 10^{-3}$, the structure expands in the $(z, v)$ plane, forming a stationary zig-zag structure. Contrary to the symmetrical case, 

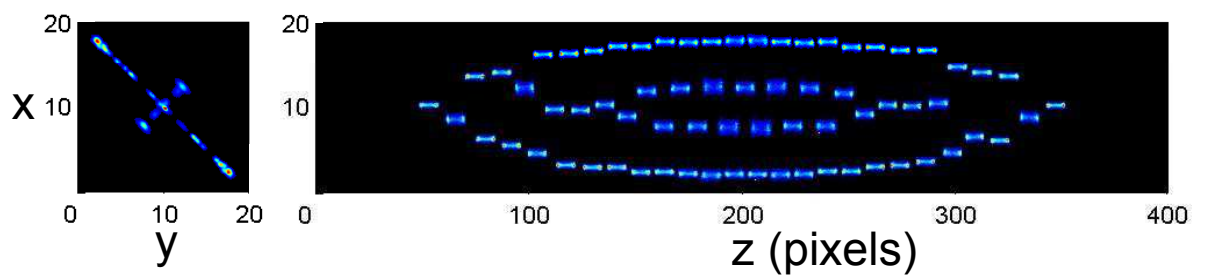

FIG. 4: (Color online) Simulated picture of 70 ions, laser cooled along the $x$-axis and trapped in a pseudo-potential with an anisotropy characterized by $\alpha=4 \times 10^{-2}$ and a non symmetric radial potential with $a_{u}=7.805 \times 10^{-3}$. The pixel size is $0.05 \mu \mathrm{m}$ in the $x$ and $y$ direction and $0.2 \mu \mathrm{m}$ in the $z$ direction. The signal associated to one pixel is proportional to the number of scattered photons in this pixel, accumulated over $2 \mathrm{~ms}$. The picture is projected on the $(x, y)$ plane (left) and on the $(y, z)$ plane (right) and the scale is in $\mu \mathrm{m}$.

the structure remains in the same plane and is not subject to chaotic rotations around the $z$-axis. Increasing $\alpha$ to $2 \times 10^{-2}$, a new $2 \mathrm{D}$ structure appears. It results from the already observed zig-zag geometry plus a central line along the $z$-axis. Increasing $\alpha$ further to $4 \times 10^{-2}$ leads to the transition of this central line to another zig-zag configuration in the $(z, u)$ plane, which is orthogonal to the outer one. A picture of such a system is shown on Fig. 4 where the different planes occupied by the two zig-zags are clearly visible. On this picture, the spot associated to each ion is elongated in the $z$ direction due to the COM motion. Intermediate values of $\alpha$ produce 3D structures without notable symmetries. For large $\alpha$ values $(\alpha \geq 30)$ corresponding to a planar structure in the symmetric case, the ions organize themselves around the $v$ axis in the $(u, v)$ plane. In the limit of very large $\alpha$ $(\simeq 800)$, the structure tends to be a perfect line along the $v$ axis.

For any $\alpha$ and any tested number of ions, the temperature ratio $T_{y} / T_{x}$ is approximately equal to 1 and does not undergo significant changes. This is attributed to the fact that the laser beam does not propagate along one of the eigen-axis of the pseudo-potential (see Eq. (4) leading to a strong coupling between the motion along $x$ and $y$. The thermal fluctuations in the radial plane are then smaller than for the symmetric case. For the same reasons, only the motion of the COM in the $z$ direction is not cooled. The $T_{z} / T_{x}$ ratio given in Fig 5 shows the same dependance with the anisotropy of the potential as in the case of a cylindrically symmetric potential. For large $\alpha$ values, the motion in the $z$ direction decouples from the other motions and the $T_{z} / T_{x}$ ratio increases. 


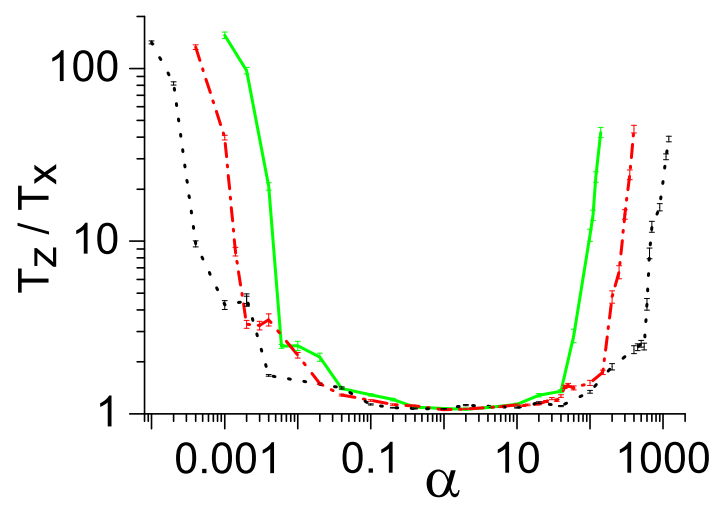

FIG. 5: (Color online) Ratio of the temperature along the trap axis over the temperature in the laser cooled direction, versus the anisotropy of the trap $\alpha$. The trapping potential is the pseudopotential with a broken cylindrical symmetry characterized by $a_{u}=7.805 \times 10^{-3}$. Calculations are made for 35 ions (solid line), 70 ions (dash-dotted line) and 140 ions (dotted line).

In the particular case of small $\alpha$ values, for which the stable configuration is a line along $z$, we observe that laser cooling induces a rotation of the ions around the $z$ axis. The combined effect of the asymmetric pseudo-potential and of the laser cooling gives birth to a rotation with an orientation depending on the polarity of the static voltage $V_{d c}$ and the axis of propagation of the laser beam, its direction being irrelevant. For a positive voltage $V_{d c}$, the maximal rotation is found when the laser is along the $x$-axis and it decreases to zero when the laser is aligned with a pair of rods of the trap. When the trap is further rotated compared to the axis of propagation of the laser, the rotation of the ions increases again but in the opposite direction. For two counter-propagating laser beams, the calculated rotation is the same if we model their mechanical effect by momentum kicks, as explained before, or if we approximate this effect by a friction force [27, 28]. When using two orthogonal laser beams, no rotation is observed.

The mean rotational frequency $\langle\omega\rangle$, calculated in the COM frame, depends on the Mathieu parameter $a_{u}$. This is illustrated on Fig. 6 for two different values of $\alpha$. For the chosen laser parameters, the two curves show a maximum for $a_{u} \simeq 10^{-4}$ and the rotation becomes negligible for $a_{u} \geq 0.01$.

The simulations presented in this section demonstrate the importance of the geometric configurations on the efficiency of the Coulomb coupling to thermalize the degrees of motion. 


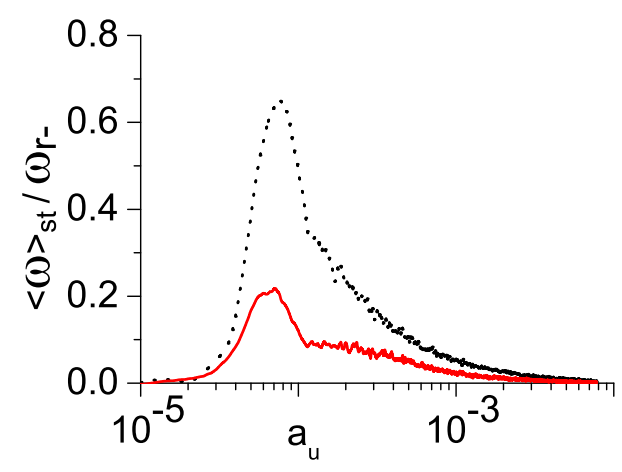

FIG. 6: (Color online) Ratio of the stationary mean rotation $\langle\omega\rangle$ over $\omega_{r-}$ of a system of 70 ions, as a function of the Mathieu parameter $a_{u}$, for an angle $\phi_{\text {rod }}=\pi / 4$ and $\alpha=10^{-3}$ (dashed line) and $\alpha=2 \times 10^{-3}$ (solid line).

In a perfect symmetric pseudo-potential, a single laser beam in the radial plane is not sufficient to cool the three degrees of freedom of a cloud of ions when its configuration does not extend in the three directions of space. Breaking the cylindrical symmetry of the potential makes a single laser beam sufficient to cool the motion in the radial plane to the Doppler limit. Nevertheless the Doppler limit may not be reached in experiments because of the rf driven motion which can lead to a heating of the sample [29, 31, 40, 41. This competition between cooling and heating can modify the efficiency of the Coulomb interaction to thermalize different degrees of freedom. Therefore, in the next section, the results obtained in the pseudo-potential approximation are compared to those obtained with the full rf electric field.

\section{IN THE RADIO-FREQUENCY ELECTRIC FIELD}

The ideal potential used in the simulations can not be reproduced in experiments because of mechanical imperfections of the trap, induced capacity, rf phase mismatch or material deposition on the electrodes [30]. Hence, dynamics related in the following for a pure radiofrequency field may not be observed in the laboratory. In the second part of this section, an additional static voltage takes into account asymmetries of the potential induced by imperfections. 


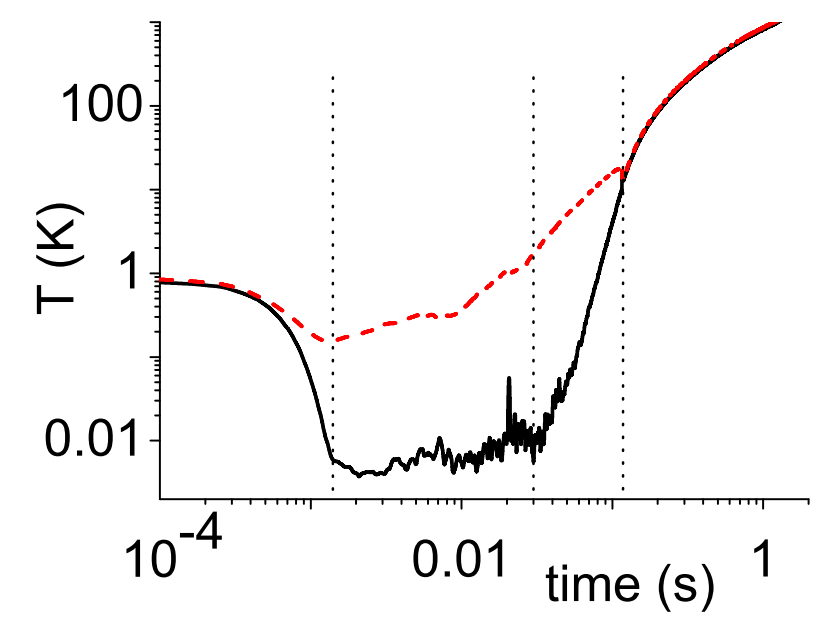

FIG. 7: (Color online) Time evolution of the temperature in the $x$ (solid line) and $y$ (dashed line) direction of motion of 70 laser cooled ions in a linear rf potential with anisotropy parameter $\alpha=7 \times 10^{-4}$. The vertical lines mark the four evolution phases mentioned in the text.

\section{A. In a pure $\mathrm{rf}$ electric field}

The Molecular Dynamics simulations of laser-cooled ions in a pure rf quadrupole field can be split into four successive temporal phases. First, the ion cloud undergoes a cooling phase until it forms a dense cloud, with a shape depending on the anisotropy of the trap. The temperatures $T_{x}$ and $T_{y}$ typically reach values between 1 and $10 \mathrm{mK}$ and an example of the time evolution of these temperatures is shown in Fig. 7. For small enough $\alpha\left(\alpha \leq 10^{-3}\right)$, for which a linear configuration along the $z$-axis is expected from [10], the amplitude of motion along $y$ is due to thermal motion and to the COM motion, as in the pseudo-potential case. But contrary to the previous case, the plane occupied by the ions during their motion is no more static. It now forms an angle with the $y$-axis oscillating at the frequency of the $\mathrm{rf}$ electric field. The introduction of a second counter-propagating laser beam suppresses these oscillations without modifying the other features described in the following.

In a second step, after being reduced by laser cooling, the amplitude of motion increases. This process is not observed in the pseudo-potential case and is attributed to rf heating [42]. In the mean time, the COM motion increases in the $y$ direction and no geometric configuration can be identified.

The third phase of the time evolution starts typically $30 \mathrm{~ms}$ after the beginning of the 


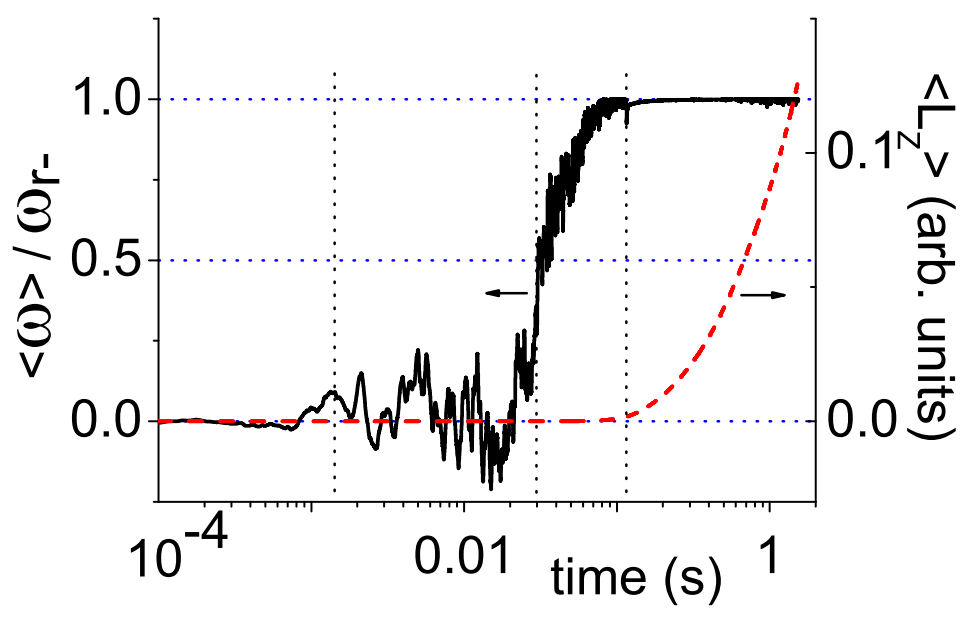

FIG. 8: (Color online) Time evolution of the ratio of the mean rotation $\langle\omega\rangle$ over $\omega_{r}$ (left axis) and angular momentum $\left\langle\bar{L}_{z}\right\rangle$ (right axis, arbitrary units), calculated in the COM frame, for a sample of 70 ions in a linear rf potential with anisotropy parameter $\alpha=7 \times 10^{-4}$. The vertical lines mark the four evolution phases mentioned in the text.

experiment with the amplification of the COM motion in the laser direction. In the ion motion in the radial plane, one can identify the rotation of the COM around the trap center, superposed to the rotation of the whole cloud around its COM (see Fig. 8). The sign of these angular frequencies is random. At the end of this third phase, the contributions of the kinetic energies associated to these two motions are of the same order.

The onset of the last part of the dynamics, taking place $0.1 \mathrm{~s}$ after the beginning of the experiment, is controlled by the mean rotation of the cloud in the COM frame $\langle\omega\rangle$. At that time, its angular frequency exactly matches the frequency of the pseudo-potential $\omega_{r}$ and remains constant. The cloud then expands in the radial direction, as illustrated on Fig. 8 where the mean angular momentum in the COM frame, $\left\langle L_{z}\right\rangle$ is plotted with $\langle\omega\rangle$.

\section{B. With an added static asymmetric potential}

We assume this particular dynamics is made possible by the perfect symmetry of the rf field and that breaking the symmetry could change the dynamics completely. To test this assumption, we add a static potential $V_{d c}$ to the rf electrodes, as for the pseudo-potential case developed in $1 \mathrm{IIB}$. When increasing $V_{d c}$, we observe an important decrease of the stationary 


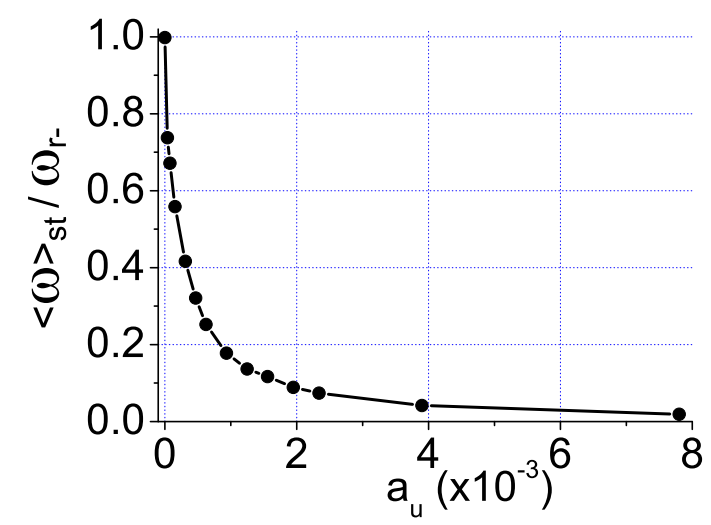

FIG. 9: Ratio of the stationary mean rotation $\langle\omega\rangle_{s t}$ over $\omega_{r-}$ of a 70 ions cloud in the COM frame, versus the Mathieu parameter $a_{u}$, in a linear rf potential with anisotropy parameter $\alpha=7 \times 10^{-4}$. mean rotation $\langle\omega\rangle_{s t}$, reported on Fig. 9. The sign of the rotation is now fixed by the polarity of the static voltage and the orientation of the laser beam, which is clockwise for positive $V_{d c}$ and a laser beam along the $x$ axis, which is consistent with the observations made in IIIB.

Figure 9 shows that for a Mathieu parameter $a_{u}=7.805 \times 10^{-3}$, corresponding to $V_{d c}=$ $20 \mathrm{~V}$, the rotation can be neglected. The dynamics reaching an equilibrium, it is then possible to calculate the temperature for the three components of motion.

As for laser cooling, the anisotropy of the trapping potential influences the rf heating rate through the spatial distribution of the ions inside the trap. Therefore, depending on the anisotropy $\alpha$, the competition between cooling and heating processes may not always lead to a cooling of the sample. The cooling rate can be tuned, to a certain extent, by the atom-laser coupling parameters introduced in IIA. Nevertheless, to focus on the effect of the geometric configurations, we keep these parameters as defined in $\amalg \mathrm{IB}$ throughout all the simulations presented here. In practice, this leads to the systematic cooling of the sample only for $\alpha$ smaller than 0.4. For larger $\alpha$, the net cooling efficiency depends on the initial conditions and increasing $\alpha$ lowers the probability to reach a cold sample, demonstrating that $\mathrm{rf}$ heating increases with $\alpha$.

To pursue the study for larger alpha values, we start with a cold cloud obtained for $\alpha=0.4$ and increase $\alpha$ slowly enough to make sure that the equilibrium is reached when the temperatures are measured. 


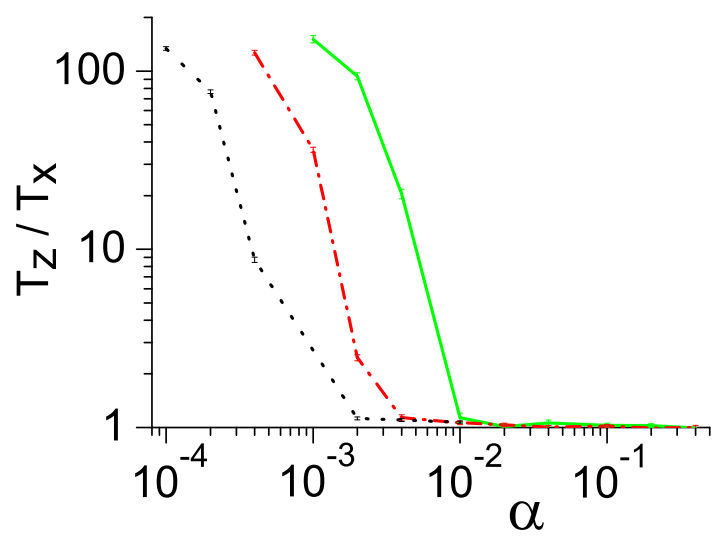

FIG. 10: (Color online) Ratio of the temperature in the uncooled direction $T_{z}$ over the temperature in the laser cooled direction $T_{x}$, versus the anisotropy of the trap $\alpha$. The trapping potential is the rf potential plus a dc contribution characterized by $a_{u}=7.805 \cdot 10^{-3}$. Calculations are made for 35 ions (solid line), 70 ions (dash-dotted line) and 140 ions (dotted line).

As for the pseudo-potential case for $a_{u} \neq 0$, the motions along $x$ and $y$ show the same temperature, but contrary to the former case, this temperature does not reach the Doppler limit and varies between 1 and $100 \mathrm{mK}$, depending on $\alpha$ and the number of ions. The temperature ratio $T_{z} / T_{x}$ is plotted on Fig. 10 for $\alpha<0.4$ and for the particular case $a_{u}=$ $7.80510^{-3}$ and shows the same behaviour as for the pseudo-potential case of Fig. 5. Indeed, the structural configurations are very similar in both cases. For $0.4<\alpha<200$ and a 70 ion cloud, the temperature ratio $T_{z} / T_{x}$ is found to be equal to one. Temperature values increase from 10 to $100 \mathrm{mK}$ when $\alpha$ is increased from 1 to 2 . They abruptly fall to $3 \mathrm{mK}$ when $\alpha$ reaches 35. This corresponds to a transition to a structure organized along the $v$ axis. When $\alpha$ reaches 200, we observe the same rise of $T_{z}$ as in the pseudo-potential case. A close look at the ion configuration reveals that this corresponds to the transition to a string along the $v$ axis.

The results of these numerical studies are twofold. First, they show that in an ideal linear rf trap, ions that are laser cooled along one radial direction can not be confined because of a rotation of the cloud leading to its expansion. Second, they show that a very small asymmetry in the radial trapping field is sufficient to inhibit this rotation and keep the ions in a stationary structure. In this case, the temperature ratio shows the same dependence with the anisotropy $\alpha$ as has been observed in the pseudo-potential. 


\section{CONCLUSION}

In this article, we study by means of Molecular Dynamics simulations, the dynamics of ions in a linear quadrupolar trap, cooled by a single laser beam propagating in the radial plane. The dependence of the thermalization of the three degrees of motion with the spatial configuration of the ions is analyzed. These simulations show the determinant role of Coulomb coupling in the thermalization process of the degrees of freedom orthogonal to the laser beam. When the trapping potential is modeled by its static pseudo-potential, we observe a strong correlation between the spatial configuration of the ions and the thermalization efficiency. Actually, the temperatures in the three directions of motion reach the same limit only if the cloud has a 3D extension. When taking into account the full rf electric field, a rotation of the cloud can be observed, that leads to its expansion and thus prevents any calculations of stationary properties. This rotation can be suppressed by breaking the symmetry of the trapping potential, which is realized by applying a static voltage to the rf electrodes. This asymmetry efficiently couples the two radial degrees of freedom which then reach the same limit temperature. When the simulations lead to cold samples, the calculated geometric structures and temperature ratios are equivalent in the pseudo-potential and in the full rf potential. In this last case, for the chosen laser cooling parameters and starting with a $1 \mathrm{~K}$ cloud, rf heating prevents to reach a cold sample when the anisotropy parameter $\alpha$ leads to a large extension of the cloud in the radial plane. Nevertheless, obtaining cold samples with high $\alpha$ values (up to 300) was made possible by increasing $\alpha$ once the sample cooled. The asymmetry required to prevent the rotation of the cloud is small enough to be encountered in any realistic experiment, which explains the possibility of stable trapping under single beam laser-cooling.

\section{Acknowledgments}

M.M. gratefully thanks Yves Elskens for very helpful and stimulating discussions. The authors would like to acknowledge fruitful discussions with Fernande Vedel and Florent 
Calvo.

[1] T. Hänsch and A. Schawlow, Optics Comm. 13, 68 (1975).

[2] D. J. Wineland and H. G. Dehmelt, Bull. Am. Phys. Soc. 20, 637 (1975).

[3] W. Paul, Rev. Mod. Phys. 62, 531 (1990).

[4] W. Neuhauser, M. Hohenstatt, P. Toschek, and H. Dehmelt, Phys. Rev. A 22, 1137 (1980).

[5] D. J. Wineland, W. M. Itano, J. C. Bergquist, and R. G. Hulet, Phys. Rev. A 36, 2220 (1987).

[6] J. C. Bergquist, W. M. Itano, and D. J. Wineland, Phys. Rev. A 36, 428 (1987).

[7] F. Diedrich, J. C. Bergquist, W. M. Itano, and D. J. Wineland, Phys. Rev. Lett. 62, 403 (1989).

[8] C. Monroe, D. M. Meekhof, B. E. King, W. M. Itano, and D. J. Wineland, Phys. Rev. Lett. 75, $4714(1995)$.

[9] D. J. Wineland and W. M. Itano, Phys. Rev. A 20, 1521 (1979).

[10] J. P. Schiffer, Phys. Rev. Lett. 70, 818 (1993).

[11] D. H. E. Dubin, Phys. Rev. Lett. 71, 2753 (1993).

[12] G. Morigi, J. Eschner, J. I. Cirac, and P. Zoller, Phys. Rev. A 59, 3797 (1999).

[13] G. Morigi and J. Eschner, Phys. Rev. A 64, 063407 (2001).

[14] G. Morigi and J. Eschner, J. Phys. B 36, 1041 (2003).

[15] J. Javanainen, Phys. Rev. Lett. 56, 1798 (1986).

[16] R. G. DeVoe, J. Hoffnagle, and R. G. Brewer, Phys. Rev. A 39, 4362 (1989).

[17] J. I. Cirac, L. J. Garay, R. Blatt, A. S. Parkins, and P. Zoller, Phys. Rev. A 49, 421 (1994).

[18] J. Pedregosa, C.Champenois, M. Houssin, and M.Knoop, Int. J. Mass Spec. 290, 100 (2010).

[19] N. McLachlan, Theory and Application of Mathieu Functions (Clarendon, Oxford, 1947).

[20] P. K. Ghosh, Ion traps (Oxford university press, 1995).

[21] M. Drewsen and A. Brøner, Phys. Rev. A 62, 045401 (2000).

[22] H. Dehmelt, Advances in Atomic and Molecular Physics 3, 53 (1967).

[23] R. Blümel, J. Chen, E. Peik, W. Quint, W. Schleich, Y. Shen, and H. Walther, Nature 334, 309 (1988).

[24] J. P. Schiffer, J. Phys. B 36, 511 (2003).

[25] S. Schiller and C. Lämmerzahl, Phys. Rev. A 68, 053406 (2003). 
[26] C. B. Zhang, D. Offenberg, B. Roth, M. A. Wilson, and S. Schiller, Phys. Rev. A 76, 012719 (pages 13) (2007).

[27] P. Lett, W. Phillips, S. Rolston, C. Tanner, R. Watts, and C.I.Westbrook, J. Opt. Soc. Am. B 6, 2084 (1989).

[28] H. Metcalf and P. van der Straten, Laser cooling and trapping (Springer, 1999).

[29] R. Blümel, C. Kappler, W. Quint, and H. Walter, Phys. Rev. A 40, 808 (1989).

[30] D. Berkeland, J. Miller, J. Bergquist, W. Itano, and D. Wineland, J. Appl. Phys. 83, 5025 (1998).

[31] V. L. Ryjkov, X. Zhao, and H. A. Schuessler, Phys. Rev. A 71, 033414 (pages 4) (2005).

[32] R. Alheit, C. Hennig, Morgenstern, F. Vedel, and G. Werth, Appl. Phys. B 61, 277 (1995).

[33] M. Vedel, J. Rocher, M. Knoop, and F. Vedel, Appl. Phys. B 66, 191 (1998).

[34] F. Vedel and J. André, Phys. Rev. A 29, 2098 (1984).

[35] S. Nosé, J. Chem. Phys. 81, 511 (1984).

[36] W. G. Hoover, A. J. C. Ladd, and B. Moran, Phys. Rev. Lett. 48, 1818 (1982).

[37] W. G. Hoover, in Statistical physics and beyond: Proceedings of $2^{\text {nd }}$ mexican meeting on mathematical and experimental physics, edited by F. Uribe, L. Garcia-Colin, and E. DiazHerrera (2005), p. 16.

[38] G. Morigi and S. Fishman, Phys. Rev. Lett. 93, 170602 (2004).

[39] G. C. Hegerfeldt and A. W. Vogt, Phys. Rev. A 41, 2610 (1990).

[40] J. D. Prestage, A. Williams, L. Maleki, M. J. Djomehri, and E. Harabetian, Phys. Rev. Lett. 66, 2964 (1991).

[41] L. Hornekær and M. Drewsen, Phys. Rev. A 66, 013412 (2002).

[42] M. Drewsen, C. Brodersen, L. Hornekær, J. S. Hangst, and J. P. Schifffer, Phys. Rev. Lett. 81, 2878 (1998). 